\title{
High-Speed Calibration and Characterization of Superconducting Quantum Processors without Qubit Reset
}

\author{
M. Werninghaus $\odot,{ }^{1,2,3, *}, \star$ D.J. Egger $\odot,{ }^{1, \dagger}$ and S. Filipp $\oplus^{1,2}$ \\ ${ }^{1}$ IBM Quantum, IBM Research-Zurich, Säumerstrasse 4, Rüschlikon 8803, Switzerland \\ ${ }^{2}$ Department of Physics, Technical University of Munich, Garching 85748, Germany \\ ${ }^{3}$ Theoretical Physics, Saarland University, Saarbrücken 66123, Germany
}

(Received 22 October 2020; revised 11 February 2021; accepted 18 March 2021; published 21 May 2021)

In order to characterize and calibrate quantum processing devices, a large amount of measurement data has to be collected. Active qubit reset increases the speed at which data can be gathered but requires additional hardware and/or calibration. The experimental apparatus can, however, be operated at elevated repetition rates without reset. In this case, the outcome of a first measurement serves as the initial state for the next experiment. Rol et al. have used this restless operation mode to accelerate the calibration of a single-qubit gate by measuring fixed-length sequences of Clifford gates that compose to $X$ gates [Phys. Rev. Appl. 7, 041001 (2017)]. However, we find that when measuring pulse sequences that compose to arbitrary operations, distortion appears in the measured data. Here, we extend the restless methodology by showing how to efficiently analyze restless measurements and correct distortions to achieve an identical outcome and accuracy as compared to measurements in which the superconducting qubits are reset. This allows us to rapidly characterize and calibrate qubits. We illustrate this bias-corrected restless measurements method by measuring a Rabi oscillation at a $250 \mathrm{kHz}$ repetition rate without any reset, for a qubit with a decay rate of $1 / 2 \pi T_{1}=3 \mathrm{kHz}$. We also show that we can measure a single- and a two-qubit average gate fidelity using randomized benchmarking 20 and 8 times faster, respectively, than measurements that reset the qubits through $T_{1}$ decay.

DOI: 10.1103/PRXQuantum.2.020324

\section{INTRODUCTION}

The characterization of quantum information processing devices [1] and the calibration of the unitary gates is a measurement-intensive task [2]. Quantum gates can be calibrated using error-amplifying gate sequences [3] and black-box optimization algorithms inspired by optimal control [4-6], which are particularly measurement intensive. Noisy quantum processors that implement Richardson error mitigation are strongly affected by the time required to calibrate and characterize the quantum gates $[7,8]$, as multiple copies of each gate, implemented with pulses of varying length, are needed. When calibrating qubit gates - for instance, with error-amplifying sequences

\footnotetext{
*max.werninghaus@gmail.com

$\dagger$ deg@zurich.ibm.com

†Present address: Walther-Meissner-Institute, WaltherMeissner-Strasse 8, 85748 Garching bei München, Germany.

Published by the American Physical Society under the terms of the Creative Commons Attribution 4.0 International license. Further distribution of this work must maintain attribution to the author(s) and the published article's title, journal citation, and DOI.
}

[3] - the most measurement-intensive task is often determination of the resulting gate fidelity, which is typically done using randomized benchmarking (RB) [9-11].

To guarantee a known and well-defined initial state, the qubits are reset to the ground state in between two consecutive measurements [12]. The easiest way to reset a qubit is by waiting for several times the decay time $\left(T_{1}\right)$ so that the energy stored in the qubit relaxes to the environment. As the $T_{1}$ times increase beyond $100 \mu \mathrm{s}$ [13], this qubit reset mechanism becomes inefficient and lengthens the time needed to acquire data. Qubit reset schemes, both conditional $[14,15]$ and unconditional [16-18] with regard to the qubit state, have therefore been developed. However, such reset mechanisms require additional hardware and/or calibration.

Qubit reset is not required to calibrate single-qubit gates, since the outcome of a projective measurement $[19,20]$ can be used as the initial state of the next operation [21]. In this operation mode, termed restless tune-up, data are gathered at elevated trigger rates, without any qubit reset. This allows data to be collected at a rate of the order of $100 \mathrm{kHz}$, limited by the pulse lengths, when characterizing a quantum processor or calibrating the unitary gates and is essential for data-intensive optimal-control schemes [6]. 
Restless measurements have been used to successfully tune up superconducting qubit gates with sequences of Clifford gates, which ideally always compose to a state flip of the qubit [21]. However, we find that when measuring pulse sequences that compose to arbitrary operations, distortion appears in the measured data. Since in restless measurements the initial state is not always the qubit ground state, the conventional data processing method that averages the measurement response does not apply $[22,23]$. Single-shot data can be used to resolve this issue. However, this requires high signal-to-noise ratios (SNRs) and the method becomes computationally expense. For instance, a singular-value decomposition (SVD) scales quadratically with the number of in-phase and quadrature (IQ) points measured.

In this paper, we show the processing steps required to efficiently analyze restless single-shot data and how to overcome the distortions to generalize the method to a broad range of experiments. We present our setup in Sec. II. In Sec. III, we use sequences of Identity $(I d)$ and NOT gates $(X)$ to show the conceptual differences between restless measurements and standard measurements. We show, in Secs. III B, how to efficiently reconstruct a restless signal and, in Secs. III C, how to process the data to avoid distortions caused by the different initial states; we therefore extend the usability of restless measurements to conventional tune-up and characterization. In Secs. IV A, we use these methods to measure a Rabi oscillation. Finally, in Secs. IV B, we show that restless measurements speed up RB. We discuss our results and draw conclusions in Sec. V.

\section{EXPERIMENTAL SETUP}

The system consists of two transmon-type fixedfrequency superconducting qubits [24] coupled by a fluxtunable coupler $[25,26]$. Experiments are carried out on one of the qubits with a transition frequency of $\omega_{01} / 2 \pi=$ $5117.22 \mathrm{MHz}$, an anharmonicity $\Delta / 2 \pi=-315.28 \mathrm{MHz}$, and coherence times of $50 \mu \mathrm{s}$ and $39 \mu \mathrm{s}$ for $T_{1}$ and $T_{2}$, respectively.

In our setup, we measure the effect of pulse sequences $k=1, \ldots, K$, each viewed as an operation $G_{k}$, with a projective measurement $M_{k}$ of the qubit. This measurement is implemented by probing a dispersively coupled readout resonator with a square pulse modulated at its resonance frequency of $6.841 \mathrm{GHz}$ [19]. The reflected signal goes through a traveling-wave parametric amplifier $[27,28]$, which allows us to perform the readout with a $2.5-\mu$ s-long pulse. After further amplification, the signal is down-converted to $\omega_{\mathrm{IF}} / 2 \pi=10 \mathrm{MHz}$ and digitized by an analog-to-digital converter (ADC), resulting in the signal $s(t)=\operatorname{Re}\left[S e^{i \theta} e^{i \omega_{\mathrm{IF}} t}\right]$, where $S$ and $\theta$ depend on the qubit state [23,29]. We represent $S$ and $\theta$ in the IQ plane as an IQ point $m=(I, Q)$ given by $I+i Q=S e^{i \theta}$.

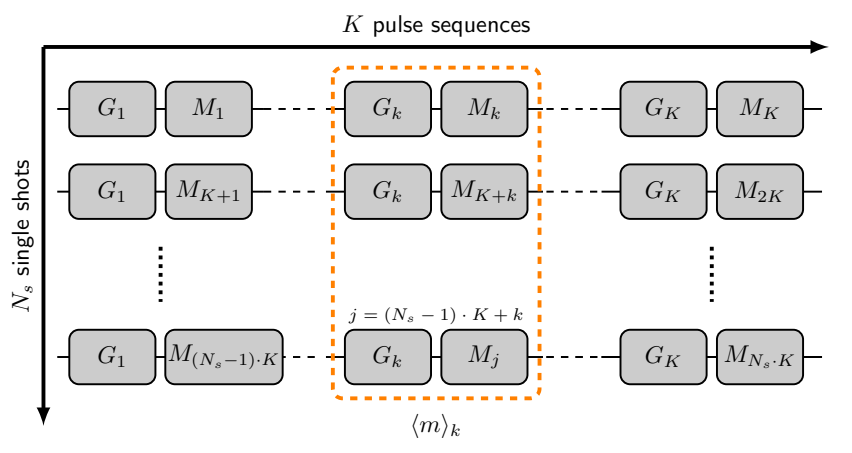

FIG. 1. An illustration of the order in which the measurements are done. We measure the effect of $K$ different quantum operations $G_{k}$, where $k=1, \ldots, K$, with a projective measurement $M_{k}$ done immediately after each $G_{k}$. All $K$ quantum operations are measured consecutively and the experiment is repeated $N_{s}$ times. When the qubit is reset in between measurements, all $N_{s}$ singleshot measurements related to $G_{k}$ can be averaged to create the average IQ point $\langle m\rangle_{k}$.

We first measure $G_{1}$ to $G_{K}$ and repeat the measurement $N_{s}$ times to gather $N_{s}$ single shots for each $G_{k}$ [30]. To keep track of time ordering, we assign an index $j$ to each measurement $M_{j}$ and its outcome $m_{j}$. For example, the $i$ th measurement of operation $G_{k}$ corresponds to measurement $M_{k+i K}$ (see Fig. 1).

When the qubit is initially in the ground state $|0\rangle, G_{k}$ creates a state $\left|\Psi_{k}\right\rangle=c_{0, k}|0\rangle+c_{1, k}|1\rangle$ with $\left|c_{0, k}\right|^{2}+\left|c_{1, k}\right|^{2}=$ 1. The measurement projects $\left|\Psi_{k}\right\rangle$ onto the ground state $|0\rangle$ or the excited state $|1\rangle$ of the qubit with probabilities $\left|c_{0, k}\right|^{2}$ and $\left|c_{1, k}\right|^{2}$, respectively [19]. Therefore, single-shot measurements form two clusters of points in the IQ plane corresponding to $|0\rangle$ and $|1\rangle$. By averaging the $N_{s}$ single-shot measurements of $\left|\Psi_{k}\right\rangle$, we obtain an IQ point,

$$
\langle m\rangle_{k}=\langle m\rangle_{\Psi_{k}}=\langle m\rangle_{|0\rangle}+\left|c_{1, k}\right|^{2}\left(\langle m\rangle_{|1\rangle}-\langle m\rangle_{|0\rangle}\right),
$$

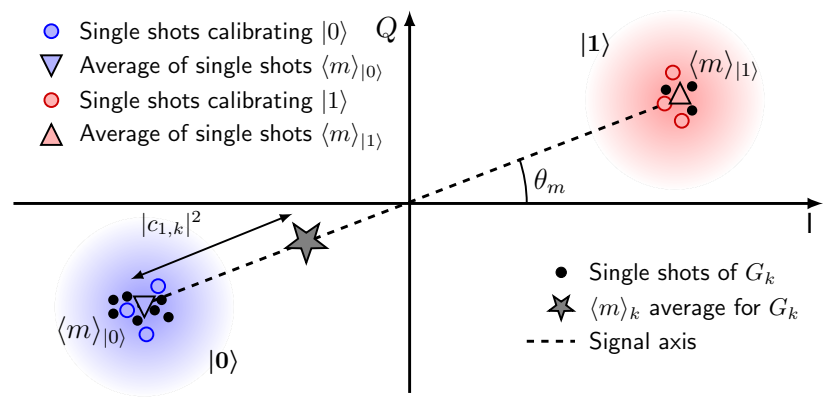

FIG. 2. An illustration of the signal axis with angle $\theta_{m}$ defined by $\langle m\rangle_{|0\rangle}$ and $\langle m\rangle_{|1\rangle}$. The IQ points form two clusters corresponding to $|0\rangle$ and $|1\rangle$. A pulse sequence $G_{k}$ that prepares the qubit in a superposition state results in single-shot IQ points (black dots) distributed between the $|0\rangle$ and $|1\rangle$ states. The average of these single shots (gray star) lies on the signal axis. 
where $\langle m\rangle_{|i\rangle}$ is the average IQ point of state $|i\rangle .\langle m\rangle_{k}$ is therefore located on the signal axis between the two IQ points $\langle m\rangle_{|0\rangle}$ and $\langle m\rangle_{|1\rangle}$. The orientation of this axis is defined by an angle $\theta_{m}$ to the in-phase axis $I$ (see Fig. 2). The distance on this axis between $\langle m\rangle_{|0\rangle}$ and $\langle m\rangle_{k}$ forms the signal of $G_{k}$, interpreted as the probability that the qubit is in state $|1\rangle$ after $G_{k}$ is applied. The signal axis is typically found by a SVD applied to the average IQ points $\langle m\rangle_{k}$.

\section{RESTLESS MEASUREMENTS}

We now illustrate the difference between restless measurements and standard measurements using a pulse sequence made of $2 N$ gates $G_{k}$, each followed by a measurement. The first $N$ gates are identity gates $\left(G_{k}=I d\right)$, while the next $N$ gates are $\pi$ rotations about the $x$ axis of the qubit Bloch sphere $\left(G_{k}=X\right)$. These $2 N$ measurements are repeated $N_{\mathrm{s}}$ times [see Fig. 3(a)]. The pulses implementing the readout and control operation $M_{j} \circ G_{k}$ are applied immediately after each trigger pulse. In the standard operation mode, the trigger rate is $R=1 \mathrm{kHz}$ to reset the qubit to the ground state by $T_{1}$ decay. Due to this

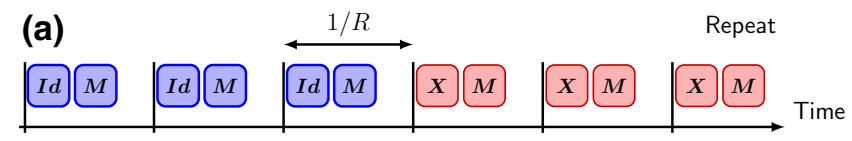

Measurements with qubit reset to $|0\rangle$
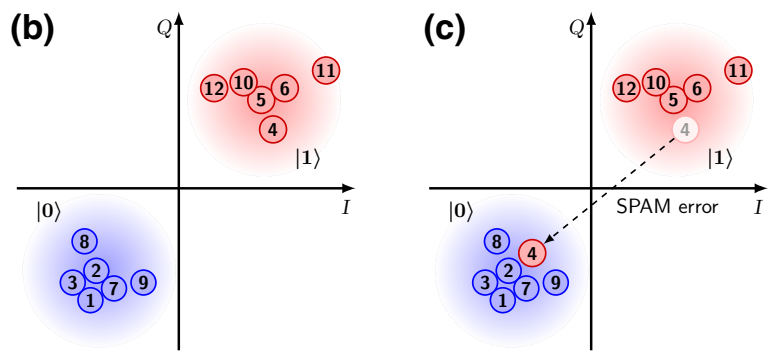

Restless measurements without qubit reset to $|0\rangle$
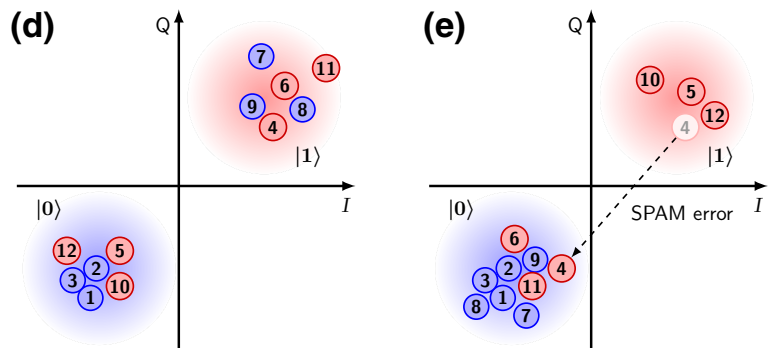

FIG. 3. An illustration of the difference between standard measurements with $T_{1}$ reset (b),(c) and restless (d),(e). The pulse sequence with $2 N=6$, shown in (a), is repeated twice. In (b)-(e), the numbers on the IQ points indicate the order in which they are acquired. The ideal outcome for standard measurements (b) is minimally affected by a SPAM error occurring at the fourth measurement (c). The ideal IQ points of restless measurements (d) are strongly affected by a SPAM error (e), which modifies all subsequent measurement outcomes. initialization, the pulse sequences $G_{k}=I d$ and $G_{k}=X$ prepare the ground and excited states of the qubit, respectively [see Fig. 3(b)]. State preparation and measurement (SPAM) errors have a minimal impact on the single shots [see Fig. 3(c)].

To perform restless measurements, we increase the trigger rate to $R=100 \mathrm{kHz}$. The qubit is not reset to its ground state after each measurement. The initial state for a given sequence $M_{j} \circ G_{k}$ is thus the outcome of the previous measurement $M_{j-1}$. As a result, the measurement outcomes of the $I d$ and $X$ operations no longer match with the ground and excited states of the qubit, respectively [see Fig. 3(d)]. Instead, they are distributed between the ground and excited states. Since a SPAM error (caused, for instance, by gate errors, $T_{1}$ decay, or unwanted qubit excitations) may change the initial state, the distribution of IQ points is randomized between the ground and excited states [see Fig. 3(e)]. Any operation $G_{k}$ that creates a superposition state will further randomize restless IQ points, as the measurement-induced projection into the ground or the excited state is a probabilistic process. These random processes are not an issue when the qubit is reset to the ground state in between measurements.

\section{A. Restless signals}

As the initial states of single-shot restless measurements of $G_{k}$ are randomized between the ground and excited states [see Fig. 3(d)], their average outcome $\langle m\rangle_{k}$ is skewed toward the origin of the IQ plane and no longer represents the centroid of the single-shot clusters [see Figs. 4(a)-4(c)]. Therefore, restless $\langle m\rangle_{k}$ do not measure the probability that $G_{k}$ excites the qubit and thus cannot be used as a signal as in Eq. (1) and Fig. 2. Instead, the state of each time-ordered single-shot measurement $m_{j}$ must be compared to the previous single shot $m_{j-1}$. We therefore assign a label $y_{j} \in\{A, B\}$ to each single shot $m_{j}$ with a discriminator to distinguish between the ground and the excited state. We can analyze restless data without knowing whether $A$ or $B$ corresponds to the qubit ground state. The restless signal is then defined as the average of an indicator function, i.e.,

$$
s_{k}=N_{s}^{-1} \sum_{i=0}^{N_{s}-1} \mathbf{1}_{k+i K},
$$

where $\mathbf{1}_{j}=1$ if $y_{j} \neq y_{j-1}$ and $\mathbf{1}_{j}=0$ otherwise [21]. Therefore, $s_{k}$ is a measurement of the probability $p_{k}$ that $G_{k}$ changes the qubit state.

To assign the labels $A$ and $B$, we need a discriminator. To avoid the computational cost of training a clustering algorithm on the two-dimensional single-shot restless IQ points $m_{j}$ (see Appendix B), we build a discriminator by projecting each $m_{j}$ on the signal axis (shown in Fig. 2), which creates a one-dimensional bimodal distribution. 

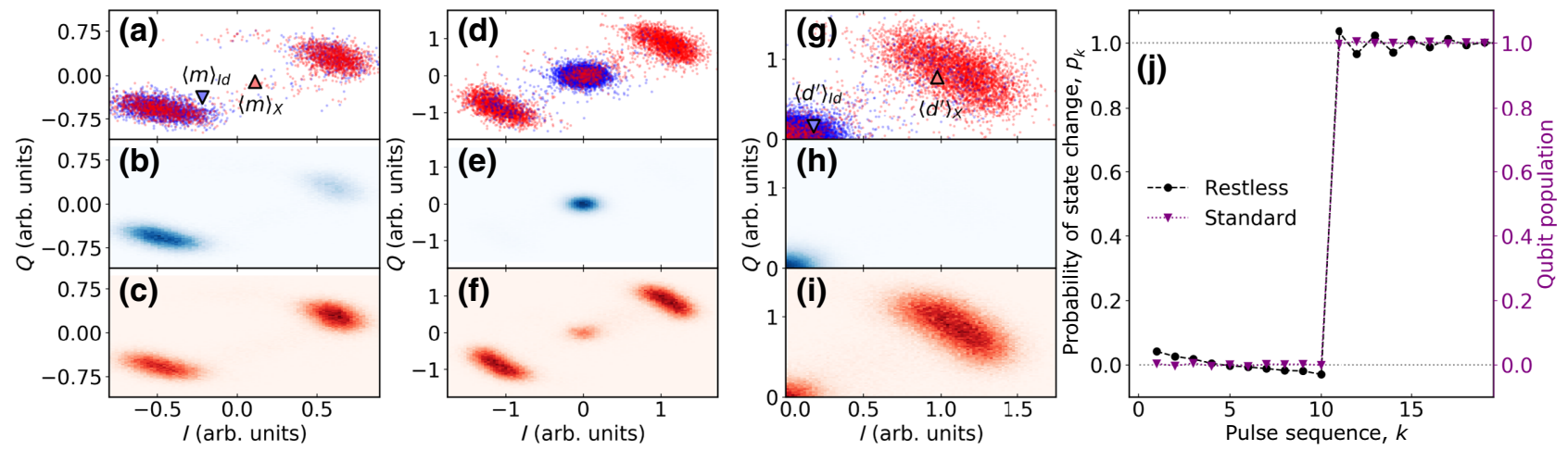

FIG. 4. Efficient reconstruction of the restless signal axis. Here, $G_{k}=I d$ for $k=1, \ldots, 10$ (blue data) and $G_{k}=X$ for $k=$ $11, \ldots, 20$ (red data). $20 \times N_{\mathrm{s}}=2 \times 10^{5}$ single-shot IQ points are measured at a $100-\mathrm{kHz}$ repetition rate. (a),(d),(g) The measured single shots $m_{j}$, the difference IQ points $d_{j}$, and the folded IQ points $d_{j}^{\prime}$, respectively. Only the first $2.5 \%$ of that data is shown to avoid overcrowding the figure. (b),(e),(h) All the single-shot data for which $G_{k}=I d$, represented as two-dimensional density plots for $m_{j}, d_{j}$, and $d_{j}^{\prime}$, respectively. (c),(f),(i) The data for $G_{k}=X$. The triangles in (a) show the average IQ points $\langle m\rangle_{I d}$ and $\langle m\rangle_{X}$ and the triangles in (g) show the average IQ points $\left\langle d^{\prime}\right\rangle_{I d}$ and $\left\langle d^{\prime}\right\rangle_{X}$. Here, $\langle\cdot\rangle_{I d}$ and $\langle\cdot\rangle_{X}$ indicate averages over experiments with $G_{k}=I d$ and $G_{k}=X$, respectively. (j) Restless signal (black dots) obtained from Eq. (4) and rescaled to [0,1] by estimating $\left\langle d^{\prime}\right\rangle_{I d}$ and $\left\langle d^{\prime}\right\rangle_{X}$ by averaging over $k=1, \ldots, 10$ and $k=11, \ldots, 20$, respectively. The purple triangles show data acquired with a 1-kHz repetition rate. Comparison of the two data sets reveals distortion in the restless data.

The discriminating line is obtained as the average of the $1 \%$ and $99 \%$ quantiles [see Fig. 5(a)].

To build our discriminator, we require the signal axis in the IQ plane that maximizes the separation of the two states. Since the averages $\langle m\rangle_{k}$ are skewed toward the origin of the IQ plane, they cannot reliably be used as input to a SVD as done in the conventional nonrestless case (see Sec. II). Indeed, estimation of the angle of a signal axis going through noisy points becomes less reliable the closer the points are to each other.

\section{B. Efficient reconstruction of restless signals}

We now show how to efficiently recover the signal axis from the single-shot restless data, which we illustrate with
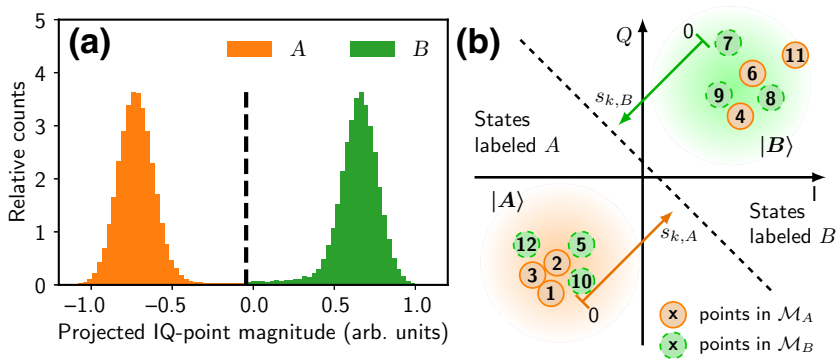

FIG. 5. (a) The one-dimensional discriminator built by projecting the $m_{j}$ IQ points onto the signal axis. The discriminating line is the average of the $1 \%$ and $99 \%$ quantiles. Since we cannot determine which peak is the ground or excited state, we assign them the labels $A$ and $B$. (b) We recolor Fig. 3(d) to illustrate which IQ points belong to $\mathscr{M}_{A}$ and $\mathscr{M}_{B}$ and indicate the signals $s_{k, A}$ and $s_{k, B}$ obtained from Eq. (7) as arrows. The dashed line is a discriminator. the same pulse sequences as described in Sec. III, with $N=10$. First, we subtract from each measured IQ point $m_{j}$ the point $m_{j-1}$ obtained in the previous measurement. The resulting difference points $d_{j}=m_{j}-m_{j-1}$ form three clusters in the IQ plane [see Fig. 4(d)]. The cluster centered around $(0,0)$ corresponds to all the measured outcomes for which $M_{j} \circ G_{k}$ does not change the qubit state, i.e., $y_{j}=y_{j-1}$. The two clusters that are not centered around $(0,0)$ are measured outcomes for which $M_{j} \circ G_{k}$ changes the qubit state, i.e., $y_{j} \neq y_{j-1}$ [see Figs. 4(e) and 4(f)]. Next, we compute the IQ points $d_{j}^{\prime}=\left|\operatorname{Re}\left(d_{j}\right)\right|+i\left|\operatorname{Im}\left(d_{j}\right)\right|$ to project the data into the first quadrant of the IQ plane, therefore combining all $M_{j} \circ G_{k}$ that change the qubit state into a single cluster. Since the $d_{j}^{\prime}$ measure a state change of the qubit (instead of the state as measured by $m_{j}$ ), the average

$$
\left\langle d^{\prime}\right\rangle_{k}=N_{s}^{-1} \sum_{i=0}^{N_{s}-1} d_{k+i K}^{\prime}
$$

is not skewed to the origin of the IQ plane as is $\langle m\rangle_{k}$. We may apply a SVD to the averaged points $\left\langle d^{\prime}\right\rangle_{k}$ to recover an axis with an angle $\theta_{d}$ in the IQ plane that yields the highest SNR. The signal axis needed for the discriminator processing the single shots $m_{j}$ has an angle $\theta_{m}$, as in Fig. 2, given by either $\theta_{d}$ or $\pi-\theta_{d}$ due to the folding $d_{j} \rightarrow d_{j}^{\prime}$ into the first quadrant. In our analysis, we try both angles and use the one with the highest SNR to build the discriminator, which allows us to reconstruct the restless signal $s_{k}$.

We observe that $\left\langle d^{\prime}\right\rangle_{k}$ is an affine transformation of $s_{k}$ since

$$
\left\langle d^{\prime}\right\rangle_{k}=\left\langle d^{\prime}\right\rangle_{I d}+p_{k}\left(\left\langle d^{\prime}\right\rangle_{X}-\left\langle d^{\prime}\right\rangle_{I d}\right)
$$


Therefore, $\left\langle d^{\prime}\right\rangle_{k}$ is a measurement of the state-change probability $p_{k}$ and is an alternative to the restless signal $s_{k}$. The end points of this axis, $\left\langle d^{\prime}\right\rangle_{I d}$ and $\left\langle d^{\prime}\right\rangle_{X}$, correspond to the $I d$ and $X$ operators. They can be measured with calibration sequences to obtain a restless signal normalized to the interval $[0,1]$, as in Eq. (2) [see Fig. 4(j)]. Additional details on the readout errors are presented in Appendix C.

\section{Correcting distortions}

We compare the restless signal to a conventional measurement of the same pulse sequences, i.e., done at a $1 \mathrm{kHz}$ repetition rate to initialize the qubit to $|0\rangle$ [see Fig. 4(j)]. The restless signal shows distortion in which identical pulse sequences produce different results. The first ten measurements, for which $G_{k}=I d$, exhibit an exponential decay and the last ten measurements, for which $G_{k}=X$, have a zigzag pattern.

At the $R=100 \mathrm{kHz}$ repetition rate, the idle time between the end of the measurement pulse and the next pulse sequence is approximately $7.5 \mu \mathrm{s}$. Measurements $m_{j}$ that yield the excited state will experience $T_{1}$ decay during this $7.5 \mu \mathrm{s}$ and will thus result in a higher probability of a SPAM error in the subsequent outcome $m_{j+1}$. Therefore, to remove this distortion, we analyze the restless data using a discriminator, as discussed in Secs. III B, and postselect each single shot according to the previous single shot to create two sets, $\mathscr{M}_{A}=\left\{m_{j} \mid y_{j-1}=A\right\}$ and $\mathscr{M}_{B}=\left\{m_{j} \mid y_{j-1}=B\right\}$. Here, $\mathscr{M}_{A}$ and $\mathscr{M}_{B}$ collect the IQ points where the previous outcome gives $A$ or $B$, respectively [see Fig. 5(b)]. Since we use pulse sequences with $I d$ and $X$ operations, we know if $m_{j}$ is obtained with $G_{k}=I d$ or $G_{k}=X$. This allows us to calculate the readout fidelities [31]

$$
\mathscr{F}_{A}=1-\frac{1}{2}\left[P_{A}(B \mid I d)+P_{A}(A \mid X)\right]=96.5 \pm 0.1 \%
$$

and

$$
\mathscr{F}_{B}=1-\frac{1}{2}\left[P_{B}(A \mid I d)+P_{B}(B \mid X)\right]=82.9 \pm 0.3 \%
$$

for $\mathscr{M}_{A}$ and $\mathscr{M}_{B}$, respectively (see Appendix C). The confidence intervals are obtained using Jeffreys' interval at a 95\% confidence level [32]. Here, $P_{x}(y \mid G)$ is the probability of measuring $y$ given the operation $G$ and the initial state $x$ and measures the SPAM error for the choices of $x, y$, and $G$ in Eqs. (5) and (6).

The different readout fidelities $\mathscr{F}_{A}$ and $\mathscr{F}_{B}$, together with the relative number of measurements in $\mathscr{M}_{A}$ and $\mathscr{M}_{B}$, create the observed distortions in the restless signal. Due to the randomization discussed in Sec. III and the $T_{1}$ decay, the probability that the single-shot measurements associated with $G_{k}$ belong, on average, to $\mathscr{M}_{A}$, labeled by $p_{k, A}$, is a function of all previous operations $G_{k}$ [see Fig. 6(a) and the model in Appendix D]. This model assumes that the $T_{1}$ time is a fit parameter. The fitted value of $T_{1}=50.0(32) \mu \mathrm{s}$ is in agreement with conventional $T_{1}$-time measurements, which further highlights that $T_{1}$ decay affects the effective readout fidelities of restless measurements. The restless signal $s_{k}$ is thus a weighted average of the signals $s_{k, A}$ and $s_{k, B}$, shown in Fig. 5(b), obtained from Eq. (2) by restricting the calculation to the IQ points in $\mathscr{M}_{A}$ and $\mathscr{M}_{B}$, respectively, i.e.,

$$
\begin{aligned}
s_{k} & =\frac{\left|\mathscr{M}_{A}\right|_{k}}{N_{s}\left|\mathscr{M}_{A}\right|_{k}} \sum_{\substack{i=0 \\
m_{k+i K} \in \mathscr{M}_{A}}}^{N_{s}-1} \mathbf{1}_{k+i K}+\frac{\left|\mathscr{M}_{B}\right|_{k}}{N_{s}\left|\mathscr{M}_{B}\right|_{k}} \sum_{\substack{i=0 \\
m_{k+i K} \in \mathscr{M}_{B}}}^{N_{s}-1} \mathbf{1}_{k+i K} \\
& =p_{k, A} s_{k, A}+\left(1-p_{k, A}\right) s_{k, B}, \quad \text { with } p_{k, A}=\frac{\left|\mathscr{M}_{A}\right|_{k}}{N_{s}} \text {. (7) }
\end{aligned}
$$

Here, $\left|\mathscr{M}_{A, B}\right|_{k}$ indicates the number of measurements belonging to $G_{k}$ that are in $\mathscr{M}_{A, B}$. This weighted average is confirmed in Fig. 6(b) which shows how the restless signal varies between $s_{k, A}$ and $s_{k, B}$ with a pattern that matches the weights $p_{k, A}$ in Fig. 6(a). We attribute the lower signal range of $s_{k, B}$ compared to $s_{k, A}$, i.e., $[13 \%, 83 \%]$ and $[2 \%, 95 \%]$, respectively, to SPAM errors (see Appendix C). More concretely, from the readout fidelities in Eqs. (5) and (6), we can now identify $B$ as the excited state, as $s_{k, B}$ has higher SPAM errors, mainly due to $T_{1}$ decay. Both signals $s_{k, A}$ and $s_{k, B}$ can be either analyzed individually or calibrated to remove SPAM errors [33] and combined into a single data set. Alternatively, retaining only the data set with the highest SNR reduces

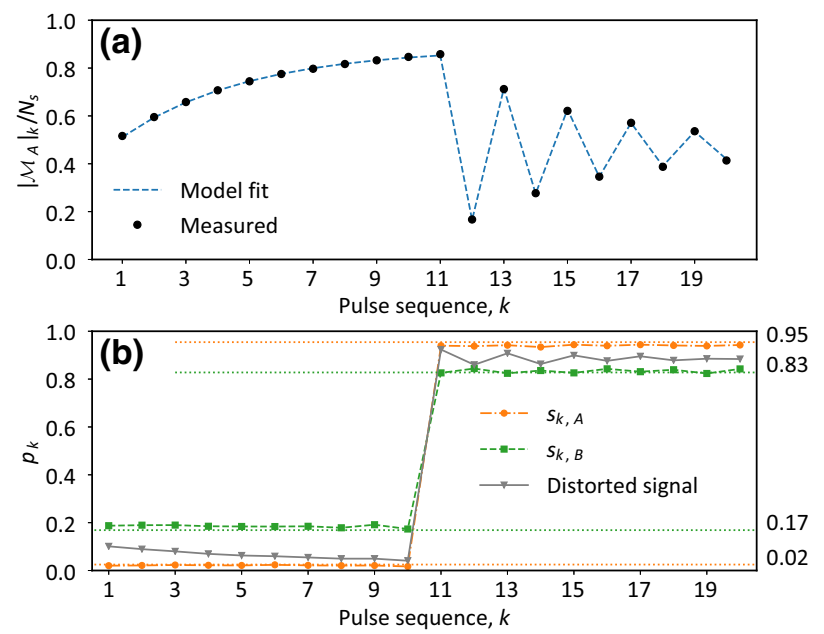

FIG. 6. (a) The probability that $m_{j} \in \mathscr{M}_{A}$, estimated by $\left|\mathscr{M}_{A}\right|_{k} / N_{\mathrm{s}}$, as a function of the measurement index. The first and last ten pulse sequences correspond to $G_{k}=I d$ and $G_{k}=X$, respectively. The dashed line is a fit to the model discussed in Appendix D. (b) The restless signals $s_{k, A}$ and $s_{k, B}$ built from $m_{j} \in$ $\mathscr{M}_{A}$ and $m_{j} \in \mathscr{M}_{B}$, respectively. The dashed lines correspond to the measured SPAM errors shown in Fig. 13. 
the number of single shots analyzed but can benefit some measurements such as RB (see Secs. IV B).

\section{RESTLESS CALIBRATION AND CHARACTERIZATION}

The characterization of quantum chips and gates requires a broad variety of measurements in which the measured behavior is fit to a function describing the expected outcome. We anticipate that restless data acquisition can speed up experiments such as spectroscopy, Ramsey, and error-amplifying gate sequences [3] for both single- and two-qubit gates. This frees up the hardware to run more quantum circuits. The signal of these experiments is often calibrated using the pulse sequences discussed in the previous sections. To illustrate calibration and characterization, we measure a Rabi oscillation and perform RB of a single- and a two-qubit gate using restless measurements.

\section{A. Rabi measurements}

To measure a Rabi oscillation between the qubit states, we apply a resonant Gaussian pulse with an amplitude $\alpha$ and subsequently measure the qubit. This pulse sequence is repeated for $K=128$ different linearly spaced values $\alpha_{k}$, starting at $-90 \%$ and ending at $90 \%$ of the maximum arbitrary-wave-form-generator output voltage of $0.8 \mathrm{~V}$. The addition of three $M \circ I d$ and three $M \circ X$ sequences allows us to mitigate readout errors by normalizing the measured signals to $[0,1]$.

We measure a Rabi oscillation by gathering data at a $1 \mathrm{kHz}$ repetition rate, averaging the IQ points $m_{j}$ over the $N_{s}=1000$ single shots, and applying a SVD to find $\theta_{m}$ (see Sec. II). This procedure reveals the Rabi oscillation nicely
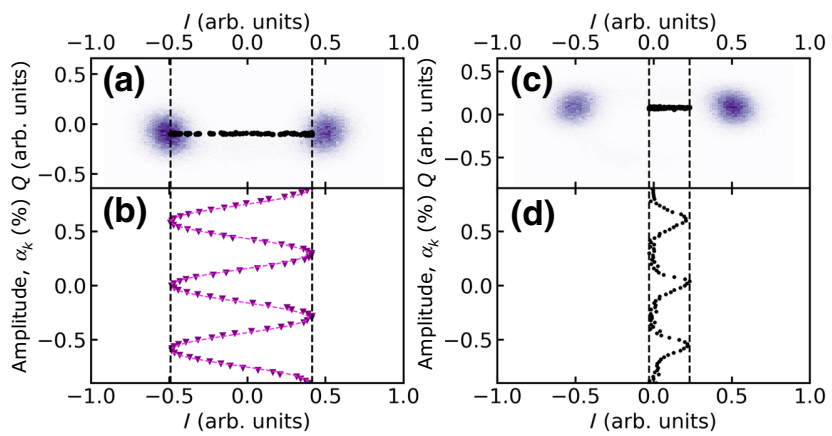

FIG. 7. The density of IQ points corresponding to a Rabi oscillation measured with a repetition rate of $1 \mathrm{kHz}$ (a) and $250 \mathrm{kHz}$ (c). A SVD applied to the average IQ points $\langle m\rangle_{k}$ yields the angle of the measurement axis $\theta_{m}$. The data are then rotated by this angle to align with the real axis for plotting. The average IQ points $\langle m\rangle_{k}$ produce the expected Rabi oscillation for standard measurements [which we fit to a cosine; see (b)] but unsurprisingly fail to reproduce the Rabi oscillation when measured at $250 \mathrm{kHz}$ [see (d)]. [see Figs. 7(a) and 7(b)]. Application of the same averaging procedure to analyze restless data, gathered at a trigger rate of $250 \mathrm{kHz}$, fails to reveal the Rabi oscillation [see Figs. 7(c) and 7(d)]. However, when we use the procedure outlined in Sec. III to build the normalized readout-errormitigated restless signals $s_{k, A}$ and $s_{k, B}$, we recover the Rabi oscillation without any distortions and in excellent agreement with the measurement at a $1-\mathrm{kHz}$ repetition rate [see Fig. 8(a)]. We combine both signals as a weighted average

$$
s_{k}=\left(\left|\mathscr{M}_{A}\right|_{k} s_{k, A}+\left|\mathscr{M}_{B}\right|_{k} s_{k, B}\right) / N_{s}
$$

which also shows the Rabi oscillation. We recover the amplitude response of the Rabi oscillation with a fit to a cosine function of the standard measurement, $s_{k, A}$, $s_{k, B}$, and $s_{k}$, yielding $0.5858(10), 0.5854(9), 0.5845(16)$, and $0.5853(10) \mathrm{rad} / \mathrm{V}$, respectively. All restless measurements reproduce the result of the standard measurement with $s_{k, B}$ slightly outside the one-standard-deviation interval. The confidence intervals reflect the standard error in the data sets shown in Fig. 8(c) and show that restless measurements have the same precision and accuracy as standard measurements. We anticipate that the methodology used here may be applied to other experiments such as Ramsey and error-amplifying gate sequences used for calibration [3].

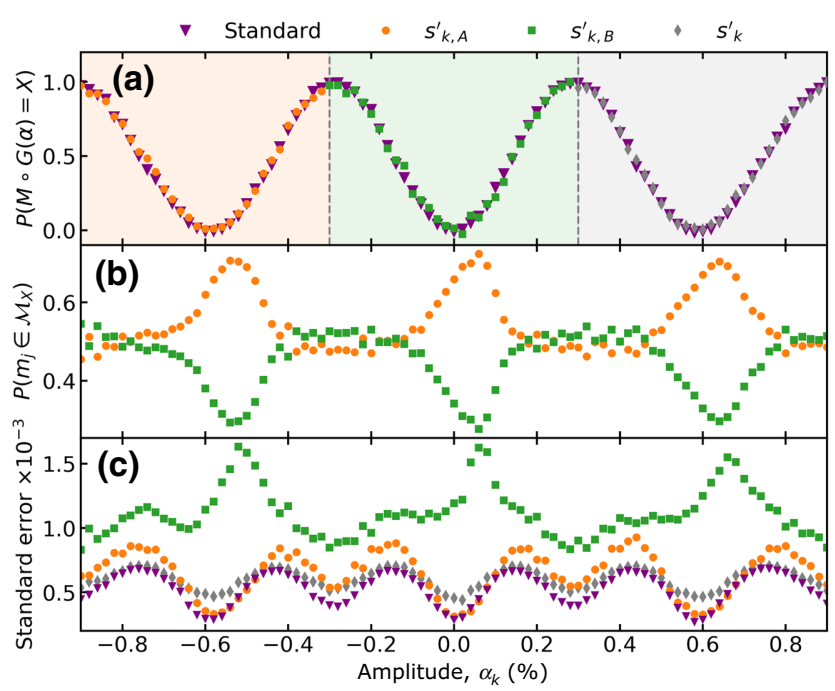

FIG. 8. (a) The restless-measured Rabi oscillation compared to the Rabi oscillation measured with a $1-\mathrm{kHz}$ repetition rate. For clarity, we only plot $s_{k, A}$ for $\alpha_{k} \in-0.9,-0.3, s_{k, B}$ for $\alpha_{k} \in$ $-0.3,0.3$, and $s_{k}$ for $\alpha_{k} \in 0.3,0.9$. (b) The measured probability that a restless IQ point belongs to $\mathscr{M}_{A}$ or $\mathscr{M}_{B}$. (c) The standard error of the standard and restless signals. Since $\mathscr{M}_{A}$ has a lower standard error, we conclude that $A$ is the ground state due to the asymmetric nature of $T_{1}$ decay. 


\section{B. Randomized benchmarking}

Randomized benchmarking requires a substantial amount of measurements [34]. Restless measurements increase the rate at which RB data are gathered. We characterize the single-qubit gates with 200 random sequences of $N_{c}$ Clifford gates, built from $X_{ \pm \pi / 2}$ and $Y_{ \pm \pi / 2}$ rotations, for 17 different values of $N_{c}$ ranging from 5 to 500 . For each Clifford sequence, we measure $N_{s}=2000$ single shots at a $1-\mathrm{kHz}$ and at a $50-\mathrm{kHz}$ repetition rate. Since each singlequbit pulse lasts $4.16 \mathrm{~ns}$, with a $4.16 \mathrm{~ns}$ buffer in between pulses, we limit the repetition rate of restless measurements to $50 \mathrm{kHz}$ to ensure that sequences with 500 Clifford gates do not overlap with the next measurement. Since our trigger rate is fixed, short Clifford sequences leave a long idle time before the start of the next sequence. $T_{1}$-induced errors of shorter Clifford sequences are thus magnified in comparison to longer Clifford sequences when the previous measurement produces a $|1\rangle$ state. We overcome this Clifford-sequence-dependent distortion by postselecting the data to use only the sequences initialized in $|0\rangle$, as they are not affected by $T_{1}$. We therefore discard $60 \%$ of the data, lowering the effective restless rate to $20 \mathrm{kHz}$. To measure the error per Clifford (EPC), we randomly select 100 of the 200 Clifford sequences and fit their mean as shown in Fig. 9(a). This procedure is repeated 1000 times to create a distribution of the measured EPC [see Fig. 9(b)]. We measure an EPC of $0.36 \pm 0.03 \%$ and $0.37 \pm 0.03 \%$ for standard and restless measurements, respectively, where the error is the standard deviation of the distribution in Fig. 9(b). From a $z$ test with $z$ value $z=(0.36-0.37) / \sqrt{0.03^{2}+0.03^{2}}=-0.25$, we conclude that both methods yield an identical EPC with an $80 \%$
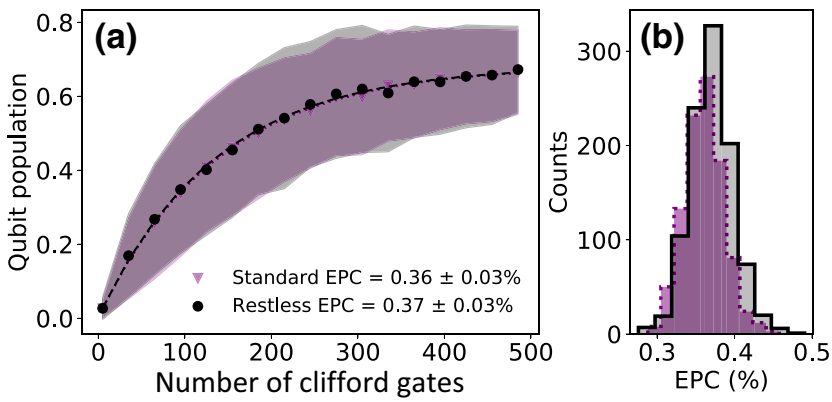

FIG. 9. Randomized benchmarking of single-qubit gates measured with a $1 \mathrm{kHz}$ and a $50-\mathrm{kHz}$ repetition rate. (a) The dots and the shaded areas show the average and the standard deviation, respectively, of all 200 realizations of random Clifford sequences. The averages are fitted to the function $A+(B-A) \alpha^{N_{c}}$, with absolute weights given by the standard error of the mean. $A, B$, and $\alpha$ are fit parameters. The measured EPC is $(1-\alpha) / 2$. (b) The distribution of 1000 measurements of the EPC. Each measurement is obtained by randomly selecting 100 of the 200 sequences and fitting the mean as in (a). The mean and standard deviation of the distribution in (b) are in the legend in (a). confidence level even after discarding $60 \%$ of the restless data.

The gathering of 6.8 million single shots takes $113.3 \mathrm{~min}$ and $2.3 \mathrm{~min}$ at $1 \mathrm{kHz}$ and $50 \mathrm{kHz}$, respectively, which emphasizes the gain of the high repetition rates. These figures do not include the constant 3 minutes needed to prepare the gate sequences, initialize the hardware, and transfer the pulse data to the arbitrary wave-form generators.

In addition to single-qubit RB, we measure the EPC of a roughly calibrated two-qubit $\mathrm{CZ}$ gate, calibrated on a different chip with an identical architecture. The $\mathrm{CZ}$ gate is implemented by modulating the tunable coupler with an oscillating magnetic flux with a frequency $\omega_{\Phi}=$ $\omega_{10}-\omega_{20}$, which drives the transition between the $|20\rangle$ and $|11\rangle$ states [34-36]. We characterize the $\mathrm{CZ}$ gate by measuring 90 random sequences of Clifford gates with up to 30 Clifford gates. Since each $\mathrm{CZ}$ gate lasts for $333 \mathrm{~ns}$, we limit the repetition rate of restless measurements to $20 \mathrm{kHz}$ to ensure that sequences with 30 Clifford gates do not overlap with the next measurement. As before, we postselect the restless data keeping only measurements initialized in $|00\rangle$ to mitigate $T_{1}$-induced errors. We therefore discard $60 \%$ of the data and the effective restless rate is $8 \mathrm{kHz}$. As before, we bootstrap the measurement by determining the EPC 1000 times, where we randomly select 50 of the 90 sequences. At $1 \mathrm{kHz}$ and $20 \mathrm{kHz}$, we measure an EPC of $6.20 \pm 0.35 \%$ and $5.99 \pm$ $0.39 \%$, respectively (see Fig. 10). From a $z$ test with $z$ value $z=(6.20-5.99) / \sqrt{0.35^{2}+0.39^{2}}=0.40$, we conclude that both methods yield an identical EPC with a $69 \%$ confidence level.
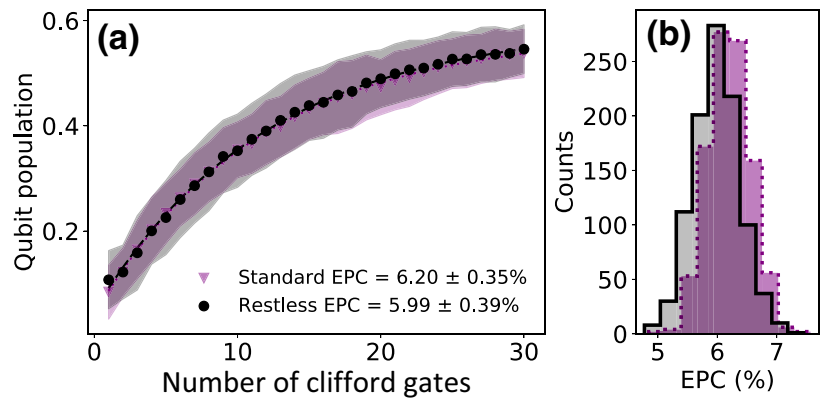

FIG. 10. Randomized benchmarking of a roughly calibrated two-qubit controlled- $Z \mathrm{CZ}$ gate measured with a $1-\mathrm{kHz}$ and a $20-\mathrm{kHz}$ repetition rate. The dots and the shaded areas show the average and the standard deviation, respectively, of 90 realizations of random Clifford sequences. The averages are fitted to the function $A+(B-A) \alpha^{N_{c}}$, with absolute weights given by the standard error of the mean. The measured EPC is $(1-\alpha) 3 / 4$. (b) The distribution of 1000 measurements of the EPC. Each measurement is obtained by randomly selecting 50 of the 90 sequences and fitting the mean as in (a). The mean and standard deviation of the distribution in (b) are in the legend in (a). 


\section{DISCUSSION AND CONCLUSIONS}

We demonstrate bias-corrected restless measurements as a method to analyze single-shot data gathered at elevated repetition rates without qubit reset by building an efficient state discriminator to correct distortions in the restless signal. Our method extends the work of Rol et al. [21] to common qubit calibration and characterization experiments by categorizing the measured single-shot data according to their initial state. By individually correcting SPAM errors in each data set, distortions in the signals can be corrected. We illustrate our method by showing that a bias-corrected restless-measured Rabi oscillation - as an exemplary measurement for calibration - and a randomized benchmarking measurement reliably reproduce the results obtained at low trigger rates. Importantly, biascorrected restless measurements can be applied without any additional requirements such as real-time analysis or extra hardware, as required for active qubit reset. In the ideal case, the effective repetition rate is limited only by the length of each individual gate sequence, including the readout pulse, which can be shortened using optimal control [6,37-39] and may require a reset of the readout resonator $[38,40]$. In our experiment, we find that the repetition rate is limited by the data-transfer rates of the data acquisition card to $250 \mathrm{MHz}$, which is only a slight reduction compared to the limit set by our readout pulse duration and a 250 -fold improvement over our standard repetition rates. The higher repetition rates of restless measurements enable more frequent and significantly faster calibration and characterization. This results in both extended up times and/or more accurately calibrated systems. While biascorrected restless measurements are generally applicable, we identify the following circumstances that need special consideration:

(1) Experiments in which the duration of the experimental sequence is comparable with the $T_{1}$ reset time, e.g., a simple $T_{1}$ measurement sequence. In this case, the repetition rate is limited by the duration of the experiment and the benefit of restless measurements is reduced.

(2) Experiments in which the duration of the gate sequence is not fixed. As the duration of the gate sequence changes, the influence of $T_{1}$-induced state preparation errors on the subset of data initialized in the excited state changes proportionally. This issue can be solved by exclusively analyzing the ground-state initialized data, as we show with randomized benchmarking, resulting in a reduction of the effective trigger rate. Alternatively, the problem can be solved by using a dynamic repetition rate such that each new sequence immediately begins after the previous readout pulse.

(3) Experiments that specifically measure the action on a particular qubit state, such as $T_{1}$ decay, where the qubit is explicitly required to be in the excited state, or algorithms, such as the quantum approximate optimization algorithm [41,42], which requires the initial state to be the ground state of the mixing operator. As above, postselection of restless data at the cost of a lower effective repetition rate is a possible solution but may not scale beyond a few qubits.

In summary, bias-corrected restless measurements can significantly speed up the calibration and characterization of superconducting qubit processors. Future work would involve analyzing the effect of the higher excited transmon states [43] and demonstrating other forms of RB $[44,45]$. Furthermore, investigation of dynamic repetition rates would improve the fidelity of restless measurements and increase the repetition rate.

\section{ACKNOWLEDGMENTS}

We thank F. Roy, S. Machnes, F. Wilhelm, M. Ganzhorn, M. Mergenthaler, P. Mueller, S. Paredes, M. Pechal, and G. Salis for insightful discussions, as well as R. Heller and H. Steinauer for technical support. We also acknowledge useful discussions and the provision of qubit devices with the quantum team at IBM T. J. Watson Research Center, Yorktown Heights. This work was supported by the (Grant No. 765267), the Intelligence Advanced Research Projects Activity (IARPA) Logical Qubits (LogiQ) program under Contract No. W911NF-16-1-0114-FE, and the Army Research Office (ARO) under Contract No. W911NF-14-1-0124.

\section{APPENDIX A: EXPERIMENTAL SYSTEM DETAILS}

The experiments presented in Secs. III B to IV A are carried out on a chip with two qubits (Q1 and Q2) at frequencies $f_{1}=5117 \mathrm{MHz}$ and $f_{2}=5745 \mathrm{MHz}$, coupled via a tunable coupler. All measurements are done on qubit Q1 using a traveling-wave parametric amplifier (TWPA) to amplify the readout signals. The readout fidelity is $96 \%$ (see Fig. 13). The amplified readout signal is digitized on an Alazar ATS9440 data acquisition card (DAC). A full schematic of all relevant components of the setup is shown in Fig. 11.

For the two-qubit measurements presented in Secs. IV B, we use a different device, as a high spurious $Z Z$ coupling prevents us from realizing a two-qubit gate on the first device. The qubit frequencies of the second device are $f_{1}=5080 \mathrm{MHz}$ and $f_{2}=6102 \mathrm{MHz}$. The readout signals are not amplified with TWPAs. The qubits are coupled by a tunable coupler with a base frequency of $f_{\mathrm{TC}}=8215 \mathrm{MHz}$, with respective coupling strengths of $g_{1}=113 \mathrm{MHz}$ and $g_{2}=143 \mathrm{MHz}$. 


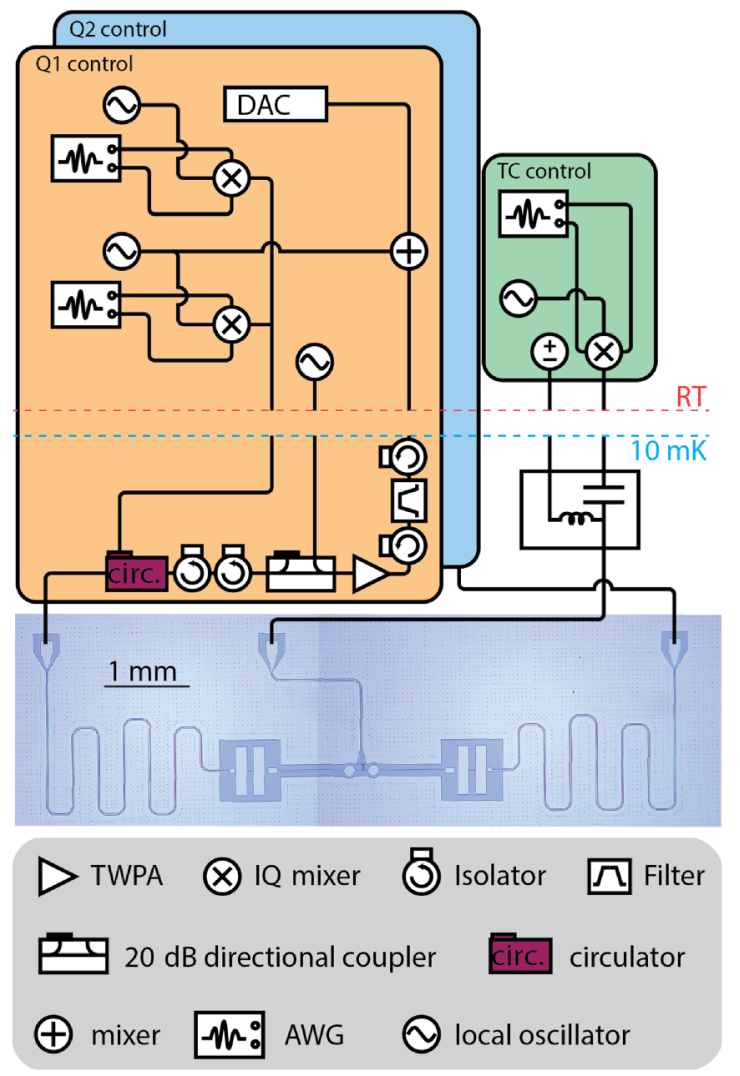

FIG. 11. A schematic of the experimental setup. The qubit control and readout pulses are generated by a Zurich Instruments HDAWG, producing the pulse envelopes modulated at $100 \mathrm{MHz}$, which are then up-converted to the qubit frequency by local oscillators (LOs). Both drives are combined and applied to the readout resonator connected to the qubit. The reflected readout signal is amplified via a traveling-wave parametric amplifier (TWPA) with a dedicated pump signal, down-converted at room temperature, and digitized. Both qubits are controlled by an identical control setup.

\section{APPENDIX B: CLUSTERING SINGLE-SHOT DATA}

Here, we compare the run time of SVD, $k$-means clustering [46], and the restless analysis in Secs. III B with a simplistic example. We numerically generate $N_{s}$ two-dimensional IQ points forming two clusters centered around $(-0.5,-0.5)$ and $(0.5,0.5)$ by sampling from a Gaussian distribution with a 0.2 standard deviation [see Fig. 12(b)]. To ensure that the example includes the number of single shots that we analyze in a typical experiment, we vary $N_{s}$ from $10^{3}$ to $10^{7}$. The SVD (from NumPy version 1.18.1) allows us to construct a signal axis (see Fig. 2), while the $k$-means classifier (from Scikit-learn version $0.21 .3)$ with $k=2$ and the restless analysis allow us to build a state discriminator [see Fig. 12(c)]. The quadratic run time of SVD [see Fig. 12(a)] makes it a poor choice when processing single-shot data. In our example, the $k$ means algorithm, based on Elkan's algorithm [47], has an
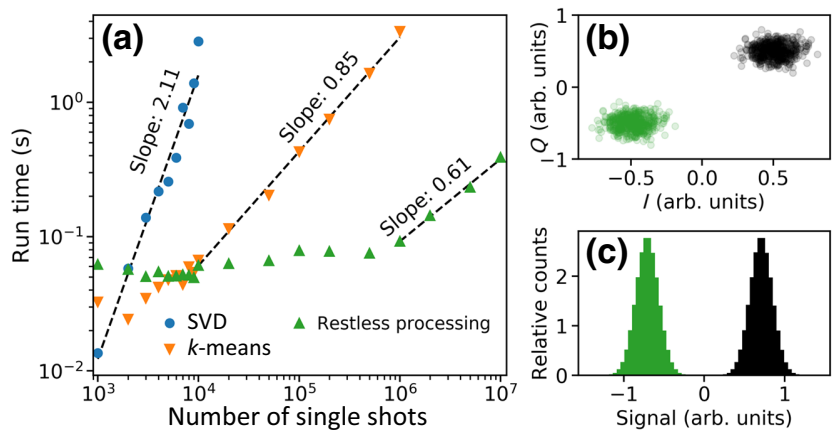

FIG. 12. (a) The run time of SVD, $k$-means, and the restless analysis in Secs. III B as a function of the number of single shots processed. The dashed lines show $\ln y=a \ln N_{s}+b$ fits used to obtain the scaling $a$ of the run time. (b) The simulated IQ data used, color coded according to the $k$-means classifier. (c) The simulated IQ data, discriminated using the restless analysis.

almost linear run time with the number of single shots. Of the three methods, the restless analysis performs best for $N_{s}>10^{4}$ [see the green triangles in Fig. 12(a)]. We expect a linear scaling $\mathscr{O}\left(N_{s}\right)$ as the number of samples increases. This simple example highlights that single shots should not be analyzed with SVD when they cannot be averaged and that the restless analysis method is fast.

\section{APPENDIX C: SPAM ERRORS}

The measurements described in Secs. III $\mathrm{C}$ allow us to quantify the SPAM errors for $\mathscr{M}_{A}$ and $\mathscr{M}_{B}$ by separating both data sets according to whether $G_{k}=I d$ or $G_{k}=X$ [see Figs. 13(a) and 13(b), respectively]. The single shots $m_{j}$, projected onto the measurement axis form a one-dimensional distribution (see the shaded curves in Fig. 13). From their cumulative distribution functions $F_{I d}$ and $F_{X}$, shown as solid lines in Fig. 13, we calculate an optimal threshold value for state discrimination given by the point where $F_{I d}$ and $F_{X}$ are maximally separated

$$
x_{T}=\underset{x}{\arg \max }\left|F_{X}(x)-F_{I d}(x)\right|,
$$

shown as a black dashed line in Fig. 13. We use $x_{T}$ to discriminate the qubit states and extract the specific SPAM error using $P_{x}(y \mid G)$ for $G \in\{I d, X\}$ and $x, y \in\{A, B\}$ (see the SPAM plots in Fig. 13).

The standard error of the difference points presented in Secs. III B is $\Delta d_{j}=\left(\Delta m_{j}^{2}+\Delta m_{j-1}^{2}\right)^{1 / 2}$ and therefore depends on two measurements. Single shots with the system initialized in the ground state $|0\rangle$ exhibit a lower SPAM-error probability due to the high assignment fidelity of the ground state and nonexistent $T_{1}$ decay. By contrast, the single shots with the system initialized in the excited state $|1\rangle$ show significantly higher SPAM errors [compare Figs. 13(b) and 13(c)]. For a readout with intrinsically low 

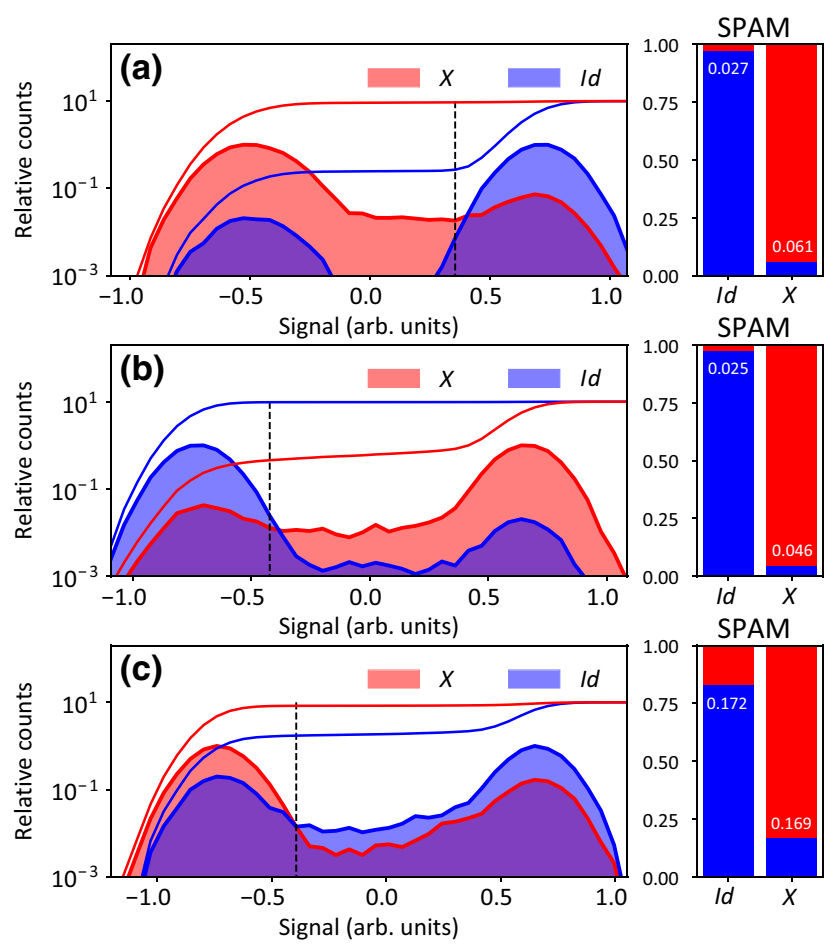

FIG. 13. The histogram of the measured IQ points for (a) standard, (b) restless $\mathscr{M}_{A}$, and (c) restless $\mathscr{M}_{B}$ measurements, split into $I d$ (blue) and $X$ (red) operations. The blue and red solid lines show the cumulative distribution functions (scaled by a factor of 10 for plotting) from which the discrimination threshold $x_{T}$, shown as a dashed black line, is obtained. The bar charts on the right show the SPAM errors $P_{A}(B \mid I d)=2.7 \%$ and $P_{A}(A \mid X)=$ $6.1 \%$ in (a), $P_{A}(B \mid I d)=2.5 \%$ and $P_{A}(A \mid X)=4.6 \%$ in (b), and $P_{B}(A \mid I d)=17.2 \%$ and $P_{B}(B \mid X)=16.9 \%$ in (c).

assignment fidelity, this effect can significantly decrease the quality of restless measurements.

\section{APPENDIX D: SIMULATION OF RESTLESS MEASUREMENTS}

Here, we build a model of restless measurements to fit the fraction of points in $\mathscr{M}_{A}$ for the data shown in Fig. 6. We model the probability $p_{|1\rangle, j}$ that measurement $j$ projects the qubit into the excited state, which depends on the previous measurements and operations, as

$$
\begin{aligned}
p_{|1\rangle, j}= & a\left[p_{|1\rangle, j-1}+\eta_{k}\left(1-p_{|1\rangle, j-1}\right)-\eta_{k} p_{|1\rangle, j-1}\right] \\
& \times e^{-1 / R T_{1}}+b .
\end{aligned}
$$

Here, $\eta_{k}$ is the probability that $G_{k}$ flips the qubit state and $\exp \left(-1 / R T_{1}\right)$ is the probability of decay due to $T_{1}$ relaxation in a time $1 / R$. $a$ and $b$ are parameters to account for SPAM errors. The probability that the qubit is projected in the excited state during the previous measurement is $p_{|1\rangle, j-1}$. The state transfer probabilities into and out of the excited state are $\eta_{k}\left(1-p_{|1\rangle, j-1}\right)$ and $\eta_{k} p_{|1\rangle, j-1}$, respectively.

We use the model in Eq. (D1) to fit $p_{k, A}$, experimentally estimated by $\left|\mathscr{M}_{A}\right|_{k} / N_{S}$, to

$$
1-\frac{1}{N_{s}} \sum_{i=0}^{N_{s}-1} p_{|1\rangle, k+i K},
$$

which assumes $A=|0\rangle$ [see Fig. 6(a)]. We set $\eta_{k}=0$ and $\eta_{k}=99 \%$ for $G_{k}=I d$ and $G_{k}=X$, respectively, and treat $T_{1}, a$, and $b$ as fit parameters. Since the qubit is in its ground state before the restless measurements begin, $p_{|1\rangle, 0}=0$, which allows us to compute the sum in Eq. (D2). This model accurately reproduces the data with fitted values $T_{1}=50.0(3.2) \mu \mathrm{s}, a=0.983(26)$, and $b=0.084(12)$ [see Fig. 6(a)].

[1] M. Devoret and R. J. Schoelkopf, Superconducting circuits for quantum information: An outlook, Science 339, 1169 (2013).

[2] P. V. Klimov, J. Kelly, J. M. Martinis, and H. Neven, The Snake Optimizer for learning quantum processor control parameters, arXiv:2006.04594 (2020).

[3] S. Sheldon, L. S. Bishop, E. Magesan, S. Filipp, J. M. Chow, and J. M. Gambetta, Characterizing errors on qubit operations via iterative randomized benchmarking, Phys. Rev. A 93, 012301 (2016).

[4] D. J. Egger and F. K. Wilhelm, Adaptive Hybrid Optimal Quantum Control for Imprecisely Characterized Systems, Phys. Rev. Lett. 112, 240503 (2014).

[5] J. Kelly, et al., Optimal Quantum Control Using Randomized Benchmarking, Phys. Rev. Lett. 112, 240504 (2014).

[6] M. Werninghaus, D. J. Egger, F. Roy, S. Machnes, F. K. Wilhelm, and S. Filipp, Leakage reduction in fast superconducting qubit gates via optimal control, npj Quantum Inf. 7, 1 (2021).

[7] K. Temme, S. Bravyi, and J. M. Gambetta, Error Mitigation for Short-Depth Quantum Circuits, Phys. Rev. Lett. 119, 180509 (2017).

[8] A. Kandala, K. Temme, A. D. Córcoles, A. Mezzacapo, J. M. Chow, and J. M. Gambetta, Error mitigation extends the computational reach of a noisy quantum processor, Nature 567, 491 (2019).

[9] E. Magesan, J. M. Gambetta, and J. Emerson, Scalable and Robust Randomized Benchmarking of Quantum Processes, Phys. Rev. Lett. 106, 180504 (2011).

[10] E. Magesan, J. M. Gambetta, and J. Emerson, Characterizing quantum gates via randomized benchmarking, Phys. Rev. A 85, 042311 (2012).

[11] A. D. Córcoles, J. M. Gambetta, J. M. Chow, J. A. Smolin, M. Ware, J. Strand, B. L. T. Plourde, and M. Steffen, Process verification of two-qubit quantum gates by randomized benchmarking, Phys. Rev. A 87, 030301 (2013).

[12] D. P. DiVincenzo, The physical implementation of quantum computation, Fortschritte der Physik 48, 771 (2000). 
[13] C. Rigetti, J. M. Gambetta, S. Poletto, B. L. T. Plourde, J. M. Chow, A. D. Córcoles, J. A. Smolin, S. T. Merkel, J. R. Rozen, G. A. Keefe, M. B. Rothwell, M. B. Ketchen, and M. Steffen, Superconducting qubit in a waveguide cavity with a coherence time approaching $0.1 \mathrm{~ms}$, Phys. Rev. B 86, 100506 (2012).

[14] D. Ristè, J. G. van Leeuwen, H.-S. Ku, K. W. Lehnert, and L. DiCarlo, Initialization by Measurement of a Superconducting Quantum Bit Circuit, Phys. Rev. Lett. 109, 050507 (2012).

[15] L. C. G. Govia and F. K. Wilhelm, Unitary-FeedbackImproved Qubit Initialization in the Dispersive Regime, Phys. Rev. Appl. 4, 054001 (2015).

[16] K. Geerlings, Z. Leghtas, I. M. Pop, S. Shankar, L. Frunzio, R. J. Schoelkopf, M. Mirrahimi, and M. H. Devoret, Demonstrating a Driven Reset Protocol for a Superconducting Qubit, Phys. Rev. Lett. 110, 120501 (2013).

[17] D. J. Egger, M. Werninghaus, M. Ganzhorn, G. Salis, A. Fuhrer, P. Müller, and S. Filipp, Pulsed Reset Protocol for Fixed-Frequency Superconducting Qubits, Phys. Rev. Appl. 10, 044030 (2018).

[18] P. Magnard, P. Kurpiers, B. Royer, T. Walter, J.-C. Besse, S. Gasparinetti, M. Pechal, J. Heinsoo, S. Storz, A. Blais, and A. Wallraff, Fast and Unconditional All-Microwave Reset of a Superconducting Qubit, Phys. Rev. Lett. 121, 060502 (2018).

[19] A. Blais, R.-S. Huang, A. Wallraff, S. M. Girvin, and R. J. Schoelkopf, Cavity quantum electrodynamics for superconducting electrical circuits: An architecture for quantum computation, Phys. Rev. A 69, 062320 (2004).

[20] A. Lupaşcu, S. Saito, T. Picot, P. C. de Groot, C. J. P. M. Harmans, and J. E. Mooij, Quantum non-demolition measurement of a superconducting two-level system, Nat. Phys. 3, 119 (2007).

[21] M. A. Rol, C. C. Bultink, T. E. O’Brien, S. R. de Jong, L. S. Theis, X. Fu, F. Luthi, R. F. L. Vermeulen, J. C. de Sterke, A. Bruno, D. Deurloo, R. N. Schouten, F. K. Wilhelm, and L. DiCarlo, Restless Tuneup of High-Fidelity Qubit Gates, Phys. Rev. Appl. 7, 041001 (2017).

[22] A. Wallraff, D. I. Schuster, A. Blais, L. Frunzio, J. Majer, M. H. Devoret, S. M. Girvin, and R. J. Schoelkopf, Approaching Unit Visibility for Control of a Superconducting Qubit with Dispersive Readout, Phys. Rev. Lett. 95, 060501 (2005).

[23] P. Krantz, M. Kjaergaard, F. Yan, T. P. Orlando, S. Gustavsson, and W. D. Oliver, A quantum engineer's guide to superconducting qubits, Appl. Phys. Rev. 6, 021318 (2019).

[24] J. Koch, T. M. Yu, J. M. Gambetta, A. A. Houck, D. I. Schuster, J. Majer, A. Blais, M. H. Devoret, S. M. Girvin, and R. J. Schoelkopf, Charge-insensitive qubit design derived from the Cooper pair box, Phys. Rev. A 76, 042319 (2007).

[25] D. C. McKay, S. Filipp, A. Mezzacapo, E. Magesan, J. M. Chow, and J. M. Gambetta, Universal Gate for FixedFrequency Qubits via a Tunable Bus, Phys. Rev. Appl. 6, 064007 (2016).

[26] M. Roth, M. Ganzhorn, N. Moll, S. Filipp, G. Salis, and S. Schmidt, Analysis of a parametrically driven exchange-type gate and a two-photon excitation gate between superconducting qubits, Phys. Rev. A 96, 062323 (2017).
[27] C. Macklin, K. O’Brien, D. Hover, M. E. Schwartz, V. Bolkhovsky, X. Zhang, W. D. Oliver, and I. Siddiqi, A near-quantum-limited Josephson traveling-wave parametric amplifier, Science 350, 307 (2015).

[28] T. Roy, S. Kundu, M. Chand, A. M. Vadiraj, A. Ranadive, N. Nehra, M. P. Patankar, J. Aumentado, A. A. Clerk, and R. Vijay, Broadband parametric amplification with impedance engineering: Beyond the gain-bandwidth product, Appl. Phys. Lett. 107, 262601 (2015).

[29] R. Bianchetti, S. Filipp, M. Baur, J. M. Fink, M. Göppl, P. J. Leek, L. Steffen, A. Blais, and A. Wallraff, Dynamics of dispersive single-qubit readout in circuit quantum electrodynamics, Phys. Rev. A 80, 043840 (2009).

[30] F. Mallet, F. R. Ong, A. Palacios-Laloy, F. Nguyen, P. Bertet, D. Vion, and D. Esteve, Single-shot qubit readout in circuit quantum electrodynamics, Nat. Phys. 5, 791 (2009).

[31] E. Magesan, J. M. Gambetta, A. D. Córcoles, and J. M. Chow, Machine Learning for Discriminating Quantum Measurement Trajectories and Improving Readout, Phys. Rev. Lett. 114, 200501 (2015).

[32] L. D. Brown, T. T. Cai, and A. DasGupta, Interval estimation for a binomial proportion, Stat. Sci. 16, 101 (2001).

[33] S. Bravyi, S. Sheldon, A. Kandala, D. C. Mckay, and J. M. Gambetta, Mitigating measurement errors in multiqubit experiments, arXiv:2006.14044 (2020).

[34] M. Ganzhorn, G. Salis, D. J. Egger, A. Fuhrer, M. Mergenthaler, C. Müller, P. Müller, S. Paredes, M. Pechal, M. Werninghaus, and S. Filipp, Benchmarking the noise sensitivity of different parametric two-qubit gates in a single superconducting quantum computing platform, Phys. Rev. Res. 2, 033447 (2020).

[35] F. W. Strauch, P. R. Johnson, A. J. Dragt, C. J. Lobb, J. R. Anderson, and F. C. Wellstood, Quantum Logic Gates for Coupled Superconducting Phase Qubits, Phys. Rev. Lett. 91, 167005 (2003).

[36] A. Bengtsson, P. Vikstål, C. Warren, M. Svensson, X. Gu, A. F. Kockum, P. Krantz, C. Križan, D. Shiri, I.-M. Svensson, G. Tancredi, G. Johansson, P. Delsing, G. Ferrini, and J. Bylander, Improved Success Probability with Greater Circuit Depth for the Quantum Approximate Optimization Algorithm, Phys. Rev. Appl. 14, 034010 (2020).

[37] D. J. Egger and F. K. Wilhelm, Optimal control of a quantum measurement, Phys. Rev. A 90, 052331 (2014).

[38] C. C. Bultink, M. A. Rol, T. E. O’Brien, X. Fu, B. C. S. Dikken, C. Dickel, R. F. L. Vermeulen, J. C. de Sterke, A. Bruno, R. N. Schouten, and L. DiCarlo, Active Resonator Reset in the Nonlinear Dispersive Regime of Circuit QED, Phys. Rev. Appl. 6, 034008 (2016).

[39] T. Walter, P. Kurpiers, S. Gasparinetti, P. Magnard, A. Potočnik, Y. Salathé, M. Pechal, M. Mondal, M. Oppliger, C. Eichler, and A. Wallraff, Rapid HighFidelity Single-Shot Dispersive Readout of Superconducting Qubits, Phys. Rev. Appl. 7, 054020 (2017).

[40] D. T. McClure, H. Paik, L. S. Bishop, M. Steffen, J. M. Chow, and J. M. Gambetta, Rapid Driven Reset of a Qubit Readout Resonator, Phys. Rev. Appl. 5, 011001 (2016).

[41] E. Farhi, J. Goldstone, and S. Gutmann, A quantum approximate optimization algorithm, arXiv:1411.4028 (2014).

[42] D. J. Egger, J. Marecek, and S. Woerner, Warm-starting quantum optimization, arXiv:2009.10095 (2020). 
[43] R. Bianchetti, S. Filipp, M. Baur, J. M. Fink, C. Lang, L. Steffen, M. Boissonneault, A. Blais, and A. Wallraff, Control and Tomography of a Three Level Superconducting Artificial Atom, Phys. Rev. Lett. 105, 223601 (2010).

[44] E. Magesan, J. M. Gambetta, B. R. Johnson, C. A. Ryan, J. M. Chow, S. T. Merkel, M. P. da Silva, G. A. Keefe, M. B. Rothwell, T. A. Ohki, M. B. Ketchen, and M. Steffen, Efficient Measurement of Quantum Gate Error by Interleaved Randomized Benchmarking, Phys. Rev. Lett. 109, 080505 (2012).
[45] A. Morvan, V. V. Ramasesh, M. S. Blok, J. M. Kreikebaum, K. O'Brien, L. Chen, B. K. Mitchell, R. K. Naik, D. I. Santiago, and I. Siddiqi, Qutrit randomized benchmarking, arXiv:2008.09134 (2020).

[46] R. Salman, V. Kecman, Q. Li, R. Strack, and E. Test, Fast $k$-means algorithm clustering, Int. J. Comput. Netw. Commun. 3, 17 (2011).

[47] C. Elkan, in Proceedings of the Twentieth International Conference on International Conference on Machine Learning, ICML'03 (AAAI Press, Washington, DC, 2003) p. 147. 